\section{Common carotid artery occlusion presenting with recurrent syncopal episodes}

\author{
Imran Kader, Steven M. Jones, \\ Catherine Harrison, Ferdinand Miteff, \\ Senthil Kumar
}

Department of Vascular Surgery, John Hunter Hospital, New Lambton, Australia

\section{Abstract}

Symptomatic common carotid artery (CCA) occlusion is an uncommon occurrence that may require surgical intervention. We aim to describe a case of CCA occlusion that presented with the unusual symptom of recurrent syncope. A 69-year-old lady presented with a history of recurrent syncopal episodes and amaurosis fugax associated with left leg weakness. She was found to have a right CCA occlusion on duplex ultrasound and angiography. She underwent a right common carotid endarterectomy and intraoperative findings revealed a heavily calcified plaque in the CCA just proximal to the bifurcation with organised thrombus filling the CCA proximally. CCA occlusion can rarely present with recurrent syncopal episodes. Surgery may be curative.

\section{Introduction}

Symptomatic occlusion of the common carotid artery (CCA) is an uncommon occurrence that may present with either acute embolic events or cerebral hypo-perfusion. ${ }^{1}$ Management does not benefit from the same extensive evidence base that surrounds disease of the internal carotid artery (ICA) and currently, surgery remains the cornerstone of treatment. We aim to describe a case of unilateral CCA occlusion in a patient who presented with the unusual symptom of recurrent syncopal episodes associated with amaurosis fugax and left leg weakness that was managed with a common carotid endarterectomy.

\section{Case Report}

A 69-year-old Caucasian lady presented to the emergency department with a three-month history of frequent falls. She was investigated by her General Practitioner for cardiac causes of her symptoms with a 24-hour ECG monitor and echocardiogram with no cause found. On further questioning she also described symp- toms of amaurosis fugax in the right eye associated with left leg weakness precluding the falls that resolved spontaneously. Her clinical examination was unremarkable. Computed tomography angiography (CTA) revealed a completely occluded right CCA (Figure 1). In the Circle of Willis (CoW) the right posterior communicating artery was patent but the contralateral Al segment of the anterior cerebral artery was absent. Duplex ultrasound demonstrated antegrade flow in the right ICA supplied by retrograde flow through the right external carotid artery (ECA) as well as a hard calcific plaque with soft thrombus proximally in the CCA (Figure 2). Aortic arch and cerebral digital subtraction angiography (DSA) was also performed (Figure 1). The DSA demonstrated patency of the proximal CCA and confirmed a short segment occlusion. The patient's anti-hypertensive medications were ceased and the case was discussed at a multidisciplinary team meeting. She proceeded to a right common carotid endarterectomy where a heavily calcified occlusive plaque was found with organised thrombus just proximal to it (Figure 3). The CCA was endarterectomised and repaired with 6-0 prolene utilising a bovine pericardial patch. The patient's antihypertensive medications were gradually reintroduced without incident on day two-post op. The patient was discharged on day three postoperatively. The patient was followed up six weeks after discharge and was completely asymptomatic.

\section{Discussion}

CCA occlusion is not encountered frequently in clinical practice. Patients with CCA occlusion most often present with recurrent TIAs from embolic phenomenon or from hypo-perfusion syndromes. ${ }^{1}$ Other rare presentations of CCA occlusion include oculomotor nerve palsy and following blunt trauma injuries to the neck. 2,3

Recurrent syncopal episodes have not been reported previously as an initial presentation of unilateral CCA occlusion. Imaging in this patient suggested that the right anterior circulation was predominantly supplied via the vertebrobasilar system through the right posterior communicating artery with a secondary contribution from the ECA/ICA. It is suspected that the absence of the Al segment of the right anterior cerebral artery in this patient made her particularly susceptible to acute hypo-perfusion secondary to the unilateral CCA occlusion. The patient's symptoms improved when her anti-hypertensive medications were ceased which supported the diagnosis of cerebral hypo-perfusion. Previous case studies have described how retrograde flow through
Correspondence: Imran Kader, Department of Vascular Surgery, John Hunter Hospital, Lookout Road, New Lambton, NSW 2305, Australia.

Tel.: +61.423.048.194 - Fax: +61.249.212. 899

E-mail: imran_kader@hotmail.com

Key words: Common carotid artery occlusion; common carotid endarterectomy; stroke; syncope and transient ischaemic attack.

Contributions: IK was the primary author and first assistant for the case operation; SJ proof read the article and contributed significantly to the discussion section; $\mathrm{CH}$ performed all of the vascular ultrasonography and interpreted the results; FM is the neurologist who initially made the diagnosis and also performed the cerebral angiograms; SK was the primary surgeon for the common carotid endarterectomy.

Conflict of interest: the authors declare no potential conflict of interest.

Received for publication: 4 August 2016.

Revision received: 24 October 2016.

Accepted for publication: 27 November 2016.

This work is licensed under a Creative Commons Attribution NonCommercial 4.0 License (CC BYNC 4.0).

CCopyright I. Kader et al., 2016

Licensee PAGEPress, Italy

Neurology International 2016; 8:6822

doi:10.4081/ni.2016.6822

the ECA can maintain antegrade flow in the ICA even in the presence of complete CCA occlusion. ${ }^{4}$ Therefore flow dynamics to cerebral hemispheres supplied by a normal CoW may be more physiological even in the presence of CCA occlusion than in cases of ICA occlusion. However patients with anatomical variants in CoW anatomy may still be symptomatic due to low cerebral perfusion pressures. ${ }^{5}$ In the current case it was thought that acute falls in blood pressure were responsible for the syncopal episodes. Although the DSA is unable to provide quantitative information on actual brain perfusion, the findings of a short segment occlusion and variant CoW anatomy made cerebral hypoperfusion the most likely diagnosis. The DSA demonstrated the extra and intracranial vasculature, as well as the pattern of collateralization. The left vertebral artery supplied the right posterior cerebral artery as well as the middle and anterior cerebral artery territories via the posterior communicating artery. Collateralized flow in the left internal carotid artery via the external carotid artery was comparatively delayed, seen on the innominate angiographic runs and contributed less to the anterior circulation flow. Formal testing of cerebral reserve such as acetazolamide challenge was not undertaken 
in the view of compelling clinical and imaging findings suggesting symptomatic cerebral hypoperfusion. Cessation of the syncopal episodes following CCA endarterectomy strongly suggests that hypoperfusion was the cause of the syncopal episodes. Operative intervention also derives a benefit in terms of stroke prevention, decreasing the risk of embolic events from the distal end of the occluded artery.

Unlike ICA disease, there are no clear treatment guidelines for CCA occlusion and data regarding the role of medical therapy is sparse. The Rile's classification of disease location may provide a useful guide in choosing the most appropriate treatment. ${ }^{6}$ The current case is an example of a Rile's classification 1A. The decision to proceed to CCA endarterectomy was based on the ability to access the entire occluded segment of artery from the standard carotid endarterectomy incision through the neck. This allowed for complete endarterectomy of the diseased segment and closure of the vessel without need for an extra-anatomical bypass. Other surgical options for Rile's type 1 disease include bypass procedures such as subclavian to carotid bypass and subclavian to ECA bypass with superficial temporal artery to middle cerebral artery bypass, or techniques such as common carotid endarterectomy. ${ }^{7}$ Rile's type 2 CCA occlusions are inoperable, except in very rare circumstances, due to the lack of distal runoff through either a patent ECA or ICA. ${ }^{6}$ Endovascular and hybrid techniques for managing CCA occlusion are still in their infancy, but may gain more popularity in the future when more specific devices are developed or with wider availability and user experience. 1 The length of the occlusion and plaque characteristics may guide the choice of technique employed. Chronic occlusions, which are often heavily calcified, and those that traverse the entire length of the CCA may be better treated by extra-anatomic bypass procedures rather than endarterectomy. Short occlusions with soft thrombus in the distal CCA may be accessed through the neck, without the need for sternotomy, lending them to local endarterectomy.

\section{Conclusions}

Unilateral CCA occlusion is rare and may be a cause of recurrent syncopal episodes. Complete cerebrovascular imaging is recommended, followed by referral to a vascular surgeon, as operative intervention may be an appropriate option.

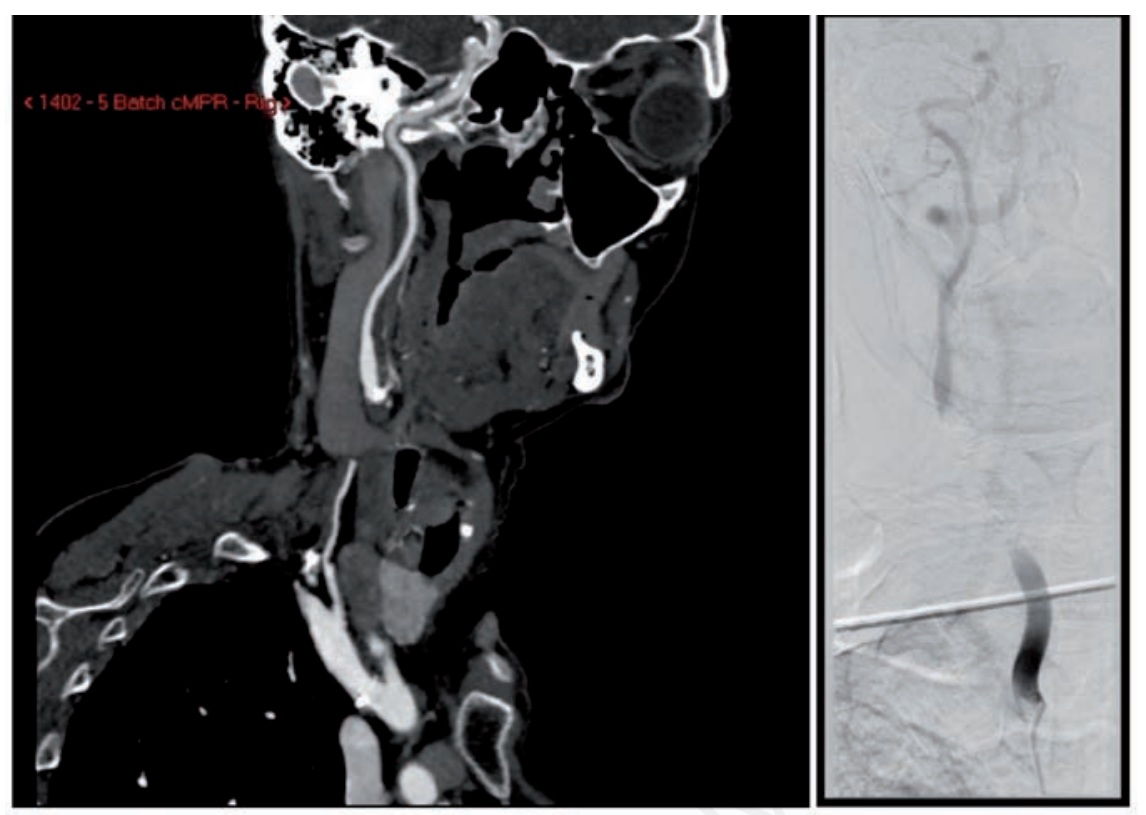

Figure 1. Computed tomography angiogram and digital subtraction angiogram demonstrating an occluded right common carotid artery and flow through the right internal carotid artery.

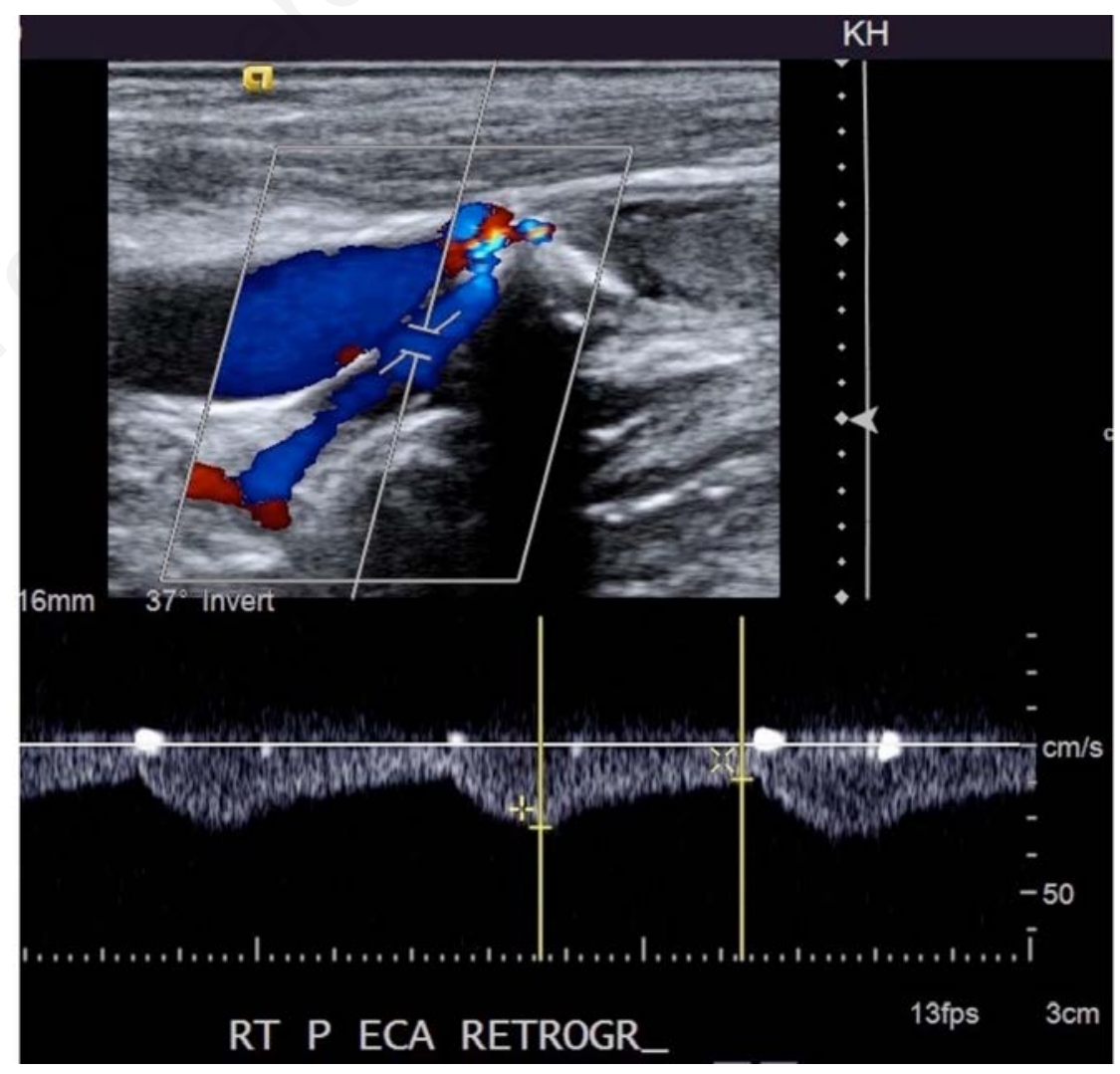

Figure 2. Duplex ultrasound demonstrating retrograde flow through the right external carotid artery that supplies antegrade flow to the right internal carotid artery. 


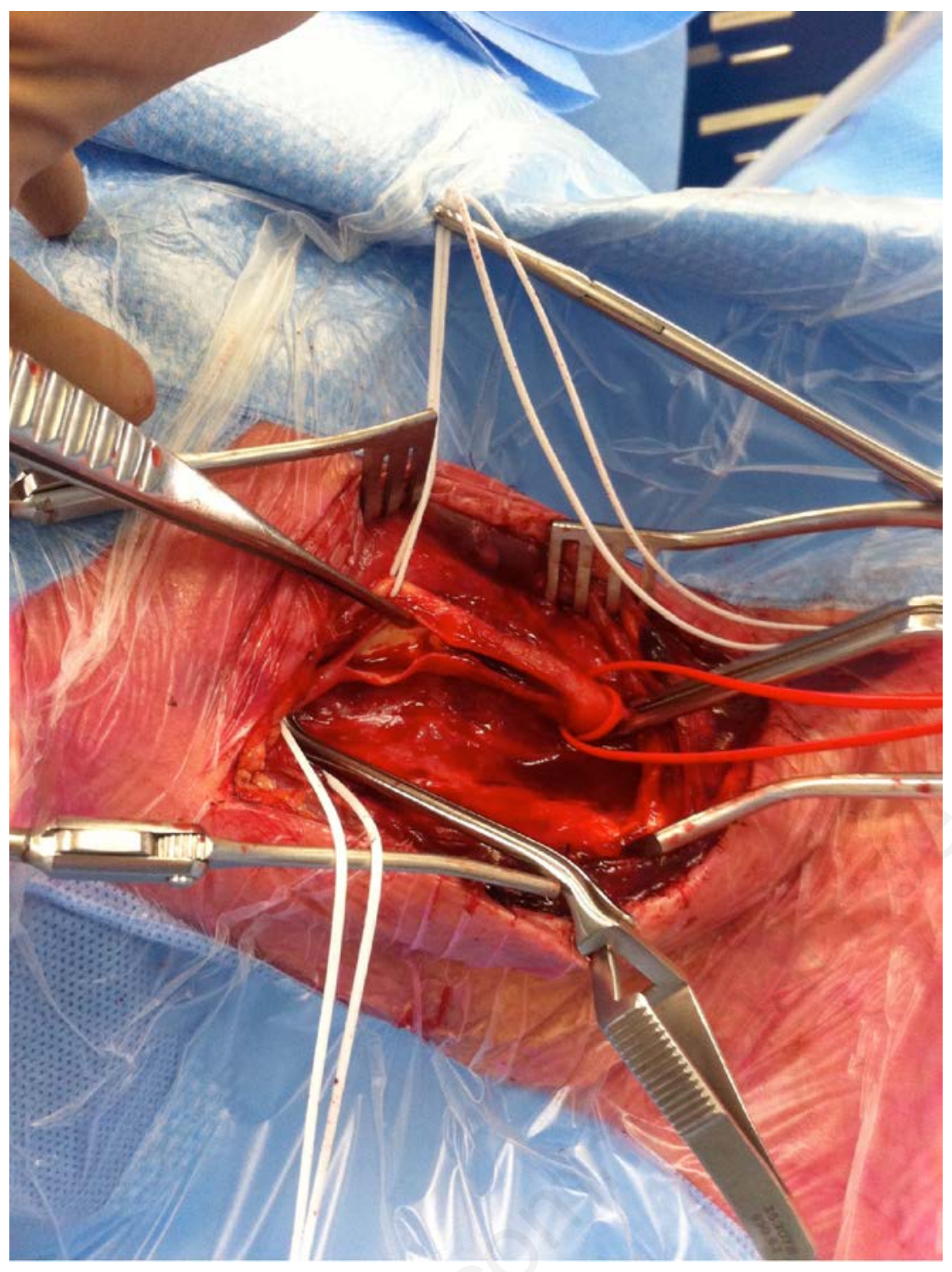

Figure 3. Intraoperative photo of the opened common carotid artery revealing a hard calcific plaque with soft thrombus.

\section{References}

1. Klonaris C, Kouvelos GN, Kafeza M, et al. Common carotid artery occlusion treatment: revealing a gap in the current guidelines. Eur J Vasc Surg 2013;46:291-8.

2. Man BK, Fu YP. Isolated oculomotor nerve palsy due to common carotid artery occlusion. BMJ Case Rep 2013;2013.

3. Perry M0, Snyder WH, Thal ER. Carotid artery injuries caused by blunt trauma. Ann Surg 1980;192:74-7.

4. Johkura K, Kuroiwa Y. Common carotid artery occlusion. Cerebrovasc Dis 2003;15:158.

5. Nakamura A, Wakugawa Y, Yasaka M, et al. Antegrade internal carotid artery collateral flow and cerebral blood flow in patients with common carotid artery occlusion. J of Ultrasound Med 2012;31:1561-6.

6. Riles TS, Imparato AM, Posner MP, Eikelboom BC. Common carotid occlusion. Assessment of the distal vessels. Ann Surg 1984;199:363-

7. Inoue T, Tsutsumi K, Adachi S, et al. Direct and primary carotid endarterectomy for common carotid artery occlusion. Report of 2 cases. Surg Neurol 2008;69:620-6. 